\title{
Civilisations
}

Revue internationale d'anthropologie et de sciences

humaines

42-2 | 1993

enQuete d'identité

\section{Étranger de tout poil ou comment on désigne l'autre}

\section{Marianne Mesnil et Assia Popova}

\section{CpenEdition \\ Journals}

Édition électronique

URL : http://journals.openedition.org/civilisations/2352

DOI : 10.4000/civilisations. 2352

ISSN : 2032-0442

\section{Éditeur}

Institut de sociologie de l'Université Libre de Bruxelles

\section{Édition imprimée}

Date de publication : 1 décembre 1993

Pagination : 179-198

ISBN : 2-87263-108-9

ISSN : 0009-8140

Référence électronique

Marianne Mesnil et Assia Popova, «Étranger de tout poil ou comment on désigne l'autre », Civilisations [En ligne], 42-2 | 1993, mis en ligne le 01 décembre 1996, consulté le 07 mai 2019. URL : http:// journals.openedition.org/civilisations/2352 ; DOI : 10.4000/civilisations.2352

Ce document a été généré automatiquement le 7 mai 2019.

(c) Tous droits réservés 


\title{
Étranger de tout poil ou comment on désigne l'autre
}

\author{
Marianne Mesnil et Assia Popova
}

« Nous arrangeons l'Histoire à la manière dont les primitifs arrangent les mythes : une manipulation arbitraire pour inventer une vision globale de

l'Univers ». Claude Levi-Strauss ${ }^{1}$

\section{Les Hommes et les autres ou le jeu des ethnonymes}

\section{Cannibales, muets et poilus ...}

1 La mosaïque ethnoculturelle de l'Europe actuelle est le résultat d'un long processus de conquêtes, de rencontres, d'oppositions entre peuples venus d'ailleurs et installés dans des régions où ils ont établi leur hégémonie sur des sociétés autochtones.

2 L'histoire de ce peuplement et de cette organisation du vieux continent est marquée par une série d'appellations ethnonymiques à travers lesquelles les divers groupements sociaux définissent leur autonomie par rapport aux autres ethnies étrangères.

3 Les termes qui désignent l'autre, c'est-à-dire l'étranger, correspondent souvent à une opposition identitaire dont la sémantique est universelle : les « hommes " (c'est-à-dire nous) et les «non hommes » (c'est-à-dire les autres). Cannibale, muet ou poilu, tels sont quelques-uns de ces appellatifs dont la caractéristique commune est de rabaisser l'autre au rang de sous-humain. C'est le cas, par exemple, du terme Samoyed qui signifie en russe " mangeur de soi-même » autrement dit « anthropophage ", nom couramment employé dans la littérature ethnographique pour désigner des ethnies sibériennes qui s'appellent elles-mêmes hommes (enets, nenets). De même, le terme Eskimo appliqué aux Inuit, signifierait mangeur de viande crue.

4 En Europe, cette opposition entre civilisé et sauvage mobilise deux figures qui expriment de manière explicite le pôle de l'animalité : c'est, d'une part, l'incapacité à maîtriser le 
langage, et d'autre part, l'absence de vêture signifiée par une pilosité abondante et apparente.

Ainsi s'explique l'étymologie du barbare balbutiant, du flamand bégayant ou de l'allemand muet.

On se rappellera en effet que le grec barbaros est un mot onomatopique qui imite le bredouillement : il signifie initialement celui qui ne parle pas le grec. ${ }^{2} C^{\prime}$ est selon une logique semblable que le français a donné le sens qu'on lui connaît au terme bégayer qui dérive de l'ancien néerlandais beggen, bavarder (en flamand beggelen)3. Quant aux Allemands. ils furent qualifiés de muets, Nemci (plur.), Nemec (sing) (prononcer nemtsi et nemets), par leurs voisins slaves qui les tenaient pour incapables de parler comme eux, un langage humain. C'est également sur ce mot slave qu'est formé l'appellatif roumain populaire neamt (plur.nemti), synonyme d'Allemand/Germain (german) ou autrichien.

De la même manière encore, l'usage du mot roumain salbatec (dérivé du latin silvaticus, « de la forêt »), se trouve précisé à propos des Valaques de l'époque de l'Empire des Assen $\left(18^{\mathrm{e}} \mathrm{s}\right.$.) (Voir plus loin) : «Soiscompréhensive et bien élevée », recommande l'Empereur Henri de Flandre à sa fille qu'il donne en mariage à un membre de cette lignée valaque, «Tu as pris un homme avec lequel tupars, qui est sauvage car tune comprends pas sa langue, ni lui la nôtré ${ }^{4}$."

8 Une manière aussi, de renvoyer l'autre à la condition d'être non humain : la faculté de parler étant le propre de l'homme, celui qui est dit muet est rejeté vers le pôle de l'animalité.

9 On pourrait sans peine allonger la liste des exemples d'ethnonymes qui procèdent d'un tel projet de dénigrement. Ainsi, la déformation occidentale de Tatar en infernal Tartare de même, la filiation étymologique entre les hérétiques bulgares bogomiles (à l'origine du mouvement cathare) et l'appellation infamante de Bou(l)gre, avec sa forme féminine bougresse qui, dès le XII ${ }^{\text {ème }}$ siècle, désignent une personne dépravée, homosexuelle ou sodomite; ou encore les termes de chrétien et crétin qui appartiennent à la même série étymologique ! L'autre, c'est donc aussi celui qui ne partage pas la même religion.

10 Et que dire des Slaves qui tentent désespérément à coups d'acrobaties linguistiques douteuses, de s'affranchir de leur réputation étymologique d'esclaves (du latin *esclavon) ${ }^{5}$ ? Pour échapper à cette filiation peu flatteuse, d'aucuns ont préféré reconnaître dans cet ethnonyme, le vocable *voda, eau, ce qui donnerait à la désignation du peuple slave le sens approximatif de Gens d'eau. D'autres linguistes (slaves bien sûr), ont même cherché une origine plus prestigieuse encore à ce terme en l'associant au vocable slava, gloire.

\section{Poilus, chevelus : les « mal rasés »}

11 Une autre manière de marquer la distance avec l'étranger, c'est d'en souligner la pilosité « animale »: celle-ci s'oppose alors au poil discipliné par le barbier, cet agent civilisateur de prime importance: armé de son rasoir, c'est lui qui fait «rentrer dans l'ordre les poilus, velus, hirsutes, et autres mal rasés $»{ }^{6}$

12 Toutefois, cette opposition entre «poilus » et « rasés » ne prend sens qu'au sein d'une relation duelle entre soi et les autres. Et la question : qui rase qui ? est ici fondamentale. Dans la mesure où l'on choisit délibérément de se (faire) raser par soi-même ou par un autre, on rejoint une communauté dont cette discipline du poil est un signe 
emblématique. Un tel comportement nous est signifié à travers un thème de facéties roumaines concernant les Tsiganes dont voici un exemple :

Un Tsigane va se faire raser pour la première fois chez le barbier; puis il se rend au cabaret pour se montrer, mais personne ne lui prête attention. Alors soulagé, il s'exclame : «Dieu soit loué! Me voilà déjà homme ! (d'après Stroescu, vol. II : 1436, n -5540)

Que l'on pense aussi par exemple à la mode romaine des cheveux courts qui s'opposent à la "Gaule chevelue " (Comata), l'indépendante Gaule celtique! Plus près de nous, les cheveux longs des haiduk ou des Klephtès du sud-est de l'Europe au siècle dernier, bandits d'honneurs et véritables "hommes des bois", hors-la-loi, marquent cette marginalisation, qui correspond ici à un acte politique volontaire.

Par contre, en Europe orientale, le rasage des esclaves (en Roumanie, ce sont généralement des Tsiganes), constitue un acte de contrainte sur le corps de l'autre, qui lui fait porter cette marque d'asservissement. Il en est de même des conscrits ou des prisonniers auxquels on signifie ainsi la discipline militaire ou carcérale. Quant aux cheveux longs et à la barbe des prêtres orthodoxes, ils permettent, au même titre que la tonsure des moines occidentaux, de distinguer ces hommes religieux du monde profane.

De telles contraintes infligées au système pileux ont également jalonné l'histoire politique des peuples. Citons-en deux exemples : celui de Pierre Le Grand qui, au XVIII ${ }^{\mathrm{e}}$ siècle, dans la foulée de sa politique d'occidentalisation de la Russie, prit des mesures draconiennes pour obliger la noblesse russe à se couper barbe et cheveux. Et celui de Napoléon Bonaparte qui lança résolument la mode des cheveux courts, mettant ainsi la noblesse française à sa solde, dans l'obligation de suivre cette mode et d'abandonner ses perruques poudrées, symbole de l'Ancien Régime.

Il y aurait ainsi à faire toute une ethnosociologie politique du poil : que l'on pense aux sociétés où la barbe est interdite (la Roumanie de Ceaucescu ou la Bulgarie de Jivkov, par exemple) ; ou à l'idéologie de la coiffure «à la brosse » des paracommando, qui s'opposait à celle des « cheveux longs » de Mai 68 etc. "Loin de tous les chacals qui portent cheveux longs " chantent aujourd'hui les jeunes militants (au poil court!) du Front National. ${ }^{7}$

\section{Tsiganes rasés et Valaques velus}

Il faut cependant nuancer le sens de l'opposition entre poilus et rasés. Chacun de ces termes peut en effet recevoir une valeur positive ou négative dans la distinction qu'il établit entre soi et les autres. Une belle illustration d'une telle portée discriminatoire du poil nous est proposée dans un petit récit étiologique bulgare, concernant deux populations étrangères: les Tsiganes et les Valaques, dont la différence, pour les Bulgares, ne tient finalement... qu'à un poil.

19 Voici le résumé de ce texte :

Un jour, le Bon Dieu se promenait sur terre accompagné de saint-Michel. Sur leur chemin, ils entendent des clameurs provenant d'une maison où se trouvent des Valaques et des Tsiganes. Intrigué par ce vacarme, saint-Michel pénètre dans la maison, et croyant à une querelle, tranche le différend en les égorgeant tous. Le Bon Dieu lui donne l'ordre d'aller les ressusciter. Mais au lieu de remettre les têtes à la bonne place, saint-Michel se trompe et replace les têtes des Tsiganes sur les corps des Valaques et inversement. Voilà pourquoi depuis ce moment, les Tsiganes se rasent la tête et les Valaques se laissent pousser les 
cheveux pour la même raison: se faire une tête «à eux ». (Réf: Texte recueilli par S. Vajtov à OIjahovo. in : SBNU 9 (1893) : 127).

\section{Des Poilus à la peau dure : Wallons, Wales, et autres Valaques}

Depuis la colonisation de la région balkano-carpathique par les Romains, les populations romanisées de cette partie de l'Europe parlant un idiome issu du latin, se désignaient elles-mêmes sous l'appellation de «Romains ». Leurs voisins slavophones - à l'Est, les actuels Russes, Biélorusses et Ukrainiens, et au Sud, les Serbes, les Croates et les Bulgares - attribuaient à ces ascendants directs des actuels Roumains le nom de Vlaxi/Vlasi ${ }^{8}$. Les Byzantins, par l'intermédiaire des Slaves vraisemblablement, ont adopté cette même appellation de provenance germanique : *Walha. Dans sa langue d'origine, elle fut utilisée pour désigner d'abord les Romains et, par extension, les populations romanisées. Les Anglo-saxons l'ont empruntée pour nommer le peuple soumis des Celtes. Ainsi cet ethnonyme anglicisé sous la forme de wealh devint un synonyme d'esclave ${ }^{9}$. Les ethnonymes Wallon (en Belgique), Wales (en Grande-Bretagne) et Valaque (Vlaxi/Vlasi) de la région Carpato-balkanique seraient donc tous des dérivations d'une appellation germanique désignant une population allogène, dominée ou insoumise.

21 La plupart de ces ethnonymes exogènes (entendez la manière dont on désigne «l'autre ») furent adoptés par les intéressés eux-mêmes; et cette naturalisation fit dès lors perdre à ces appellatifs toute trace d'accentuation péjorative.

Tel ne fut pourtant pas le cas du terme Valaque. Couramment utilisé jusqu'au début de ce siècle par les peuples balkaniques, à l'exception des Roumains eux-mêmes, le vocable Valaque dans ses multiples réalisations locales désigne une population de pasteurs nomades pratiquant la transhumance, à l'instar des Sarakatsanes grécophones, avec lesquels ils furent et restent encore souvent confondus en Bulgarie ${ }^{10}$.

La raison de cet usage généralisé du terme Valaque pour désigner des bergers transhumants, réside dans le fait que l'élevage extensif était l'occupation principale de l'une des branches importantes de cette ethnie au parler latin : elle sillonnait d'un bout à l'autre la Péninsule balkanique, depuis la plaine danubienne jusqu'aux montagnes grecques du Pinte et de la Chalcidique, en passant par le faîte de la chaîne montagneuse des Balkans. ${ }^{11}$

De nos jours, l'ethnonyme Valaques a été partout supplanté par le terme endogène «Roumains» (Români., du latin Romani) qui a été utilisé de tous temps par ces populations pour se désigner. ${ }^{12}$

Pour les Roumains, qui n'utilisent jamais le terme pour se désigner eux-mêmes,vlah (fém. vlaha) ou valah, (plur.valahi), existe cependant, mais avec la signification de nom donné aux Roumains au Moyen Age, par d'autres peuples (Dict. Breban).

26 A cette même série, se rattache encore le terme Vlax qui désigne les Tsiganes de Paris (venus de Roumanie).

27 Ce double système d'appellatifs fonctionne aussi dans la toponymie : pour l'étranger, la région habitée par les populations "valaques ", était bien sûr la "Valachie ». Mais pour ces Roumains alias Valaques, il s'agissait de "Tsara Romaneasca», littéralement, « le Pays roumain », également désigné par le terme de Tsara Munteneasca ou Muntenia, c'est-à-dire "pays des montagnes $»^{13}$. Quant aux deux autres provinces historiques habitées par cette même ethnie, rune, appelée localement «Ardeal », fut connue dans l'histoire sous le nom 
latin de Transilvania, littéralement pays « d'au-delà des montagnes » et l'autre reçut le nom de Moldova (Moldavie), mot de la toponymie slave adopté par les Roumains euxmêmes. Mais les Bulgares désignèrent également cette région Bogdanie du nom de son fondateur présumé, Bogdan (Voir plus loin). Valaques (en Transylvanie) et Moldaves (en Moldavie) en sont les populations de langue roumaine. En outre, les Roumains/Valaques du sud des Carpates reçurent parfois l'appellation de rumàn, forme médiévale qui prenait alors le sens de "tributaire, corvéable» («mais jamais esclave») ${ }^{14}$. Toutefois dans le discours usuel des ethnies voisines, l'appellation traditionnelle exogène de Valaques subsiste en tant que doublet de l'appellation officielle, un doublet-surnom quelque peu familier et teinté de connotations péjoratives. ${ }^{15}$

\section{Des nomades boiteux}

28 Cette attitude méprisante des voisins balkaniques à l'égard de tels Valaques est encore plus fortement marquée à l'égard des Aroumains (Aromîni en langue roumaine) ${ }^{16}$, que les Grecs, les Serbes et les Bulgares qualifient de «Valaques boiteux »: Kutsovlax(s)i.

Les populations aroumaines formaient jadis une société pastorale nomade à la démographie importante, dans l'ensemble de la péninsule balkanique ${ }^{17}$.Peu à peu sédentarisés et assagis par rapport à leur réputation d'antan, ces anciens "rôdeurs hirsutes" sont aujourd'hui disséminés en petits groupements compacts parmi les populations slavophones, grécophones et arvanites de la Macédoine ${ }^{18}$. Au sujet de l'origine de leur surnom peu flatteur, on raconte l'histoire suivante :

Au temps de la domination ottomane, les jeunes gens valaques, afin de se soustraire au service militaire obligatoire ordonné par le sultan, faisaient semblant de boiter, d'où le sobriquet de "valaques boiteux " qui leur est encore appliqué de nos jours. (cf. Archives de Tsepenkov).

\section{Velus ou tondus : mythologie à rebrousse-poil}

\section{Un mythe bulgare sur « les autres »: les « Valaques velus »}

Mais l'image des bergers boiteux ne peut égaler l'accumulation de traits négatifs que met en scène à leur propos un récit étiologique bulgare au ton quelque peu facétieux. Le mythe relate en effet l'histoire de l'expulsion des Valaques hors du pays et de leur relégation dans un territoire situé au nord du Danube. Le récit mythique s'appuie sur l'association homophonique entre le terme vlas (sing.)/vlasi (pluriel) qui signifie « poil(s), cheveu(x) » et l'ethnonyme vlax/vlasi (Valaque). De cette homophonie, en l'occurrence accidentelle ${ }^{19}$,surgit la possibilité de traduire l'ethnonyme Vlasi comme «Gens veluspoilus $»^{20}$.

31 Le jeu étymologique, facétieux à souhait, mis en oeuvre par le mythe, n'est en effet nullement gratuit. Car nous pensons, contrairement à Monsieur de Saussure, que dans les associations mobilisées par les productions mythologiques, la quête de sens précède l'heureuse rencontre des homophonies. ${ }^{21}$ Ainsi, si les Bulgares n'avaient sous la main des homophones convenables pour faire des Roumains des sauvages velus, ils auraient $\mathrm{pu}$ trouver bien d'autres cheminements symboliques pour faire de leurs voisins, comme tout homme qui se respecte, des sauvages incultes. En réalité, dans le cas de l'étymologie populaire, ce n'est pas l'homophonie qui produit du sens mais tout au contraire, ce sont 
les significations préexistantes qui servent à créer, improviser, inventer de la mythologie à ses dépens.

Voici donc le texte provenant du village d'Orjaxovo situé sur le Danube en Bulgarie du Nord-ouest $\mathrm{t}^{22}$. Nous l'avons quelque peu abrégé mais en avons respecté les tournures spécifiques et le style de la fabulation:

I

Jadis, au temps des Latins, apparurent dans le royaume, des malfaiteurs qui tuaient et volaient. Les gens demandèrent au Tsar de les débarrasser de ces bandits. Mais celui-ci n'en fit rien parce que les bandits étaient des Latins comme lui. Mais ils finirent par dépasser les bornes, et le Tsar ordonna qu'on les conduise dans l'actuel territoire de la Valachie, sans leur donner ni bétail, ni nourriture, ni femmes - rien, si ce n'est un chat comme bête de transport et de trait pour labourer la terre. Ils durent pêcher le poisson du Danube et en faire commerce, pour se nourrir. Le Tsar leur interdit également de construire des maisons et ils durent creuser des trous dans la terre qu'ils recouvraient de feuilles de maïs et de roseaux. Isolés pendant des siècles, ils devinrent sauvages à tel point qu'ils en oublièrent leur langue et ne purent plus prononcer qu'un seul mot: «o-o-o-pre-chte! $»^{23}$.Ces hommes ensauvagés ne reconnaissaient ni Tsar, ni évêque; ils ne payaient d'impôts à personne et n'allaient pas à l'église, parce qu'ils n'avaient aucune foi. Et ils ne se coupaient pas les cheveux, ne se rasaient pas, et comme ils se promenaient presque nus, leur corps se couvrit de poils, comme des bêtes! C'est pourquoi les gens commencèrent à les appeler « Vlasi », c'est-à-dire poilus, chevelus.

II

Un jour, le Tsar bulgare qui était en deçà du Danube, eut pitié d'eux et décida de les christianiser et de les apprivoiser. Il leur envoya des popes pour les baptiser, mais ils allaient se cacher dans leurs trous, dans les roseaux. Le Tsar envoya alors des soldats qui attrapèrent tous les « Vlasi » et les baptisèrent. Il ordonna ensuite de les transférer en deçà du Danube pour qu'ils s'y établissent quelques années, le temps d'apprendre à parler. Mais ils n'arrivaient pas à bien assimiler le bulgare, qu'ils continuèrent à confondre avec leur ancienne langue. Quand les Vlasi furent quelque peu apprivoisés, le Tsar les renvoya au-delà du Danube en leur enjoignant de lui payer un tribut, afin qu'ils n'oublient pas la foi ; et il les obligea à revenir chaque année à Pâques pour chercher du pain consacré. ${ }^{24}$

\section{Entre Carpates et Danube : un vide historique où s'abîme le mythe}

Pour comprendre ce récit, recueilli vers la fin du XIX e siècle, il faut sans doute se rappeler la tradition de passé étatique dont il témoigne.

En effet, dès le $7^{\mathrm{e}}$ siècle, dans les Balkans, les khans et autres princes, bientôt mués en tsars, c'est-à-dire empereur (César) bulgares, amorcèrent le processus d'unification d'une population multiethnique et multilingue en lui imposant en même temps une religion supra-ethnique, une langue officielle (le parler de leurs sujets slavophones majoritaires) et une écriture élaborée pour son évangélisation : le cyrillique ${ }^{25}$. Les seuls à rester rebelles à cette politique impériale normalisante et centralisatrice, furent les Valaques ${ }^{26}$. Et il est sans doute significatif qu'aucune des Chroniques bulgares ou grecques n'aient mentionné les frontières boréales de l'Etat, alors que la Valachie faisait pourtant partie des deux royaumes bulgares successifs ( $\mathrm{du} 7^{\mathrm{e}} / 11^{\mathrm{e}}$ siècles, puis $\mathrm{du} 12^{\mathrm{e}} / 14^{\mathrm{e}}$ siècles) : jusque dans l'historiographie officielle, la Valachie transdanubienne apparait comme une zone nébuleuse et obscure. 
Dans notre texte aussi, nous avons affaire à une géographie mythique où l'ailleurs sauvage, malfamé et mal connu, sorte de goulag médiéval, se situe toujours, pour les Balkaniques, dans le désert de Minuit ${ }^{27}$.

Pourtant nous savons bien aujourd'hui, que ces contrées qualifiées de désertiques, en réalité, ne l'étaient pas. Mais l'absence d'une volonté politique centralisatrice des populations qui y étaient établies a sans doute permis au mythe des sociétés historiques d'investir ces espaces pour y instaurer les limites de leur Etat, rejetant dès lors ces autochtones dans les marécages de la sauvagerie.

37 La dimension mythique «disqualifiante» du récit fondateur est ici évidente. Tous les traits de rabaissement que nous avons rencontrés dans la typologie des ethnonymes rappelée plus haut, s'y retrouvent en effet dans une redondance significative : ne pas avoir de langue, ni de religion, ni de loi ; se nourrir étrangement (sans agriculture ni élevage); ou encore habiter "sous terre ", ne pas se vêtir, et finalement, être poilu : c'est ce dernier trait de l'animalité qui donne sens à tout le récit et fonde l'étymologie populaire bulgare de cette population étrange et voisine.

38 Il s'agit une fois de plus de "mettre à distance " une population dont le genre de vie constitue une sorte de déviance, une menace par rapport au reste du système social étatique: dans notre texte, il est question d'une population des bords du Danube, stigmatisée comme pêcheurs qui ne savent ni semer ni labourer. Mais on sait combien le problème est universel: la société des agriculteurs sédentaires se trouve toujours provoquée par les populations plus ou moins mobiles. que ce soit des pasteurs transhumants ou nomades qui traversent leur territoire. La menace de désordre que porte en soi cette différence se trouve ici conjurée par le rejet de la population non conforme ...

\section{Et le chat ?}

9 Sans vouloir nous attarder sur ce qui apparaît dans notre récit comme un détail surréaliste, le thème du "chat laboureur » nous indique cependant deux pistes de recherches : la première rejoint directement la question ici soulevée des ethnonymes. On trouve en effet dans des textes de facture byzantine, l'indication d'une " zoomorphie des peuples » dont Hasdeu fait remonter l'origine au XII ${ }^{e}$ siècle. A la question : «quelles sont les particularités des langues?", chaque peuple se voit attribué un nom d'animal : l'Italien est un lion, l'Allemand un aigle, le Turcun dragon, ... et le Roumain est... un chat. ${ }^{28}$

Nous retrouvons par ailleurs notre chat, à nouveau «laboureur» dans des motifs de facéties roumaines qui visent également à ironiser sur la différenciation ethnique : mais il s'agit cette fois des rapports entre Roumains et Juifs. ${ }^{29}$

\section{Un mythe hongrois sur « les autres » : Les Roumains tonsurés}

41 Il n'est pas qu'en Bulgarie que les populations voisines se voient gratifiées d'une mythologie «à rebrousse-poil» lorsqu'il s'agit de leur donner une origine. L'historiographie roumaine contient un récit, sans doute de source hongroise, sur l'ascendance peu glorieuse des populations du Maramures. Il s'agit d'un texte publié vers 1650 par le Roumain Simion Dascalul ${ }^{30}$. La comparaison avec le récit bulgare sur les Vlasi 
indique le même mécanisme mythique de «mise à distance » dépréciative de ses voisins «trop proches ». En voici le résumé31

Jadis, les Tatares habitaient la région de Moldavie; sous le règne de Laslau (c'est-à-dire Vladislav), seigneur hongrois, ils envahirent la Transylvanie. Le Prince se rendit alors auprès de l'Empereur de Rome afin d'obtenir de l'aide pour venir à bout de ces ennemis. L'empereur y consentit en ces termes : il met à la disposition du Prince tous les bandits qui remplissent les prisons de Rome, à condition toutefois qu'il s'engage à ne pas les lui ramener. C'est ainsi que Laslau s'en revint avec une bande de malfaiteurs qu'il eut soin de marquer avant de les incorporer dans son armée, « en leur brûlant les cheveux sur le pourtour de la tête, signe qui s'est maintenu jusqu'à nos jours dans le pays de Moldavie et de Maramures où l'on se tond tout autour de la tête $\aleph^{32}$. Une fois les Tatares refoulés audelà du Mont Rodna ${ }^{33}$ grâce à ce renfort, les Hongrois craignant de pâtir de cette population de "brigands romains ", décidèrent de les installer ailleurs : "c'est pourquoi ils leur ont choisi un endroit désert et sauvage, entouré de montagnes entre le pays polonais et le pays hongrois, qui s'appelle aujourd'hui Maramures (...) là où ils vivent encore de nos jours ». (in Bràtianu :267)

On retrouve donc ici les thèmes déjà rencontrés dans le récit bulgare, tels que :

- A l'origine, les populations allogènes (roumaines) ne sont autres que des bandits dont un Etat organisé a voulu se débarrasser.

- Pour ce faire, on les a envoyé ailleurs, sur des territoires voisins mais déserts. C'est là qu'ils se sont installés et qu'ils se trouvent toujours.

- Et surtout, ces populations ont une « marque » du système pileux qui les distingue des autres en signifiant leur asservissement : si les Vlasi de Bulgarie s'ensauvagent au point de ne plus se raser et de se couvrir de poils, c'est l'inverse qui se passe chez les anciens bandits de l'Empire romain : on leur rase la tête afin qu'ils portent la tonsure et soient dès lors reconnaissables avant d'être incorporés dans l'armée hongroise.

Il importe peu pour notre propos que cette tonsure ait réellement été pratiquée comme sembleraient l'indiquer certaines informations ${ }^{34}$. Le rapprochement des deux récits étiologiques sur les "voisins velus ou tondus » indique à souhait la fonction de cette distinction dans les textes en question ${ }^{35}$.

\section{Le motif de la « descente » (« descalecat ») : Un mythe roumain sur «soi »}

Tout autre est bien sûr le regard que l'on porte sur soi-même! Autant le récit mythique exogène a-t-il soin de disqualifier l'autre, autant le miroir que l'on se tend à soi-même se complaît-il à rehausser de prestige notre image, à travers celle de nos ancêtres, ce qui ne manque pas d'augmenter encore la distance qui nous sépare de nos voisins désormais rejetés dans l'ailleurs de la sauvagerie ou de la barbarie. Ainsi sont nés sur la terre de la mythologie roumaine, aux confins de son historiographie, des récits de fondations aux résonances universelles. Tel ce thème du descalecat, véritable "cheval de bataille » des historiens roumains : l'étymologie elle-même de ce terme en suggère déjà la dimension mythique. En effet, si l'expression de descalecat est utilisée dans le sens de "fondation d'état ", son sens étymologique et premier signifie "descente de cheval»; et les historiens veulent y voir l'idée plus métaphorique de « descente de la montagne ». Ainsi distinguent-ils trois grandes "descentes» (ou Fondations d'État) dans l'histoire roumaine : la première sans doute, du point de vue chronologique, est celle du Voïvode de 
Transylvanie Radul Negru, le Prince Noir, « descendu » de Fagaras (sud des Carpates) pour fonder le Pays de Muntenia ou Tsara Romàneasca, autrement dit, la Valachie ${ }^{36}$. Suivent deux autres descentes, qui correspondent à la fondation de la Moldavie : la plus célèbre est celle du Prince Dragos, venu du Maramures et qui, à l'issue d'une chasse à l'aurochs, décide de fonder l'État moldave ${ }^{37}$. Elle est bientôt suivie d'une seconde descente du Maramures, celle du Prince Bogdan, révolté contre le roi hongrois Ludovic I. Une autre figure de Bodgan (le même ?), a également suscité, côté bulgare, l'appellation de Bogdanie (Bogdansko) pour désigner la Moldavie.

Enfin, moins connue (pour des raisons idéologiques évidentes) que les autres mythes de Fondations d'Etats roumains, une version slavisante retiendra pourtant notre attention: elle est due au même Simion Dascalul décidément dépourvu de préoccupations nationalistes (qui seraient d'ailleurs quelque peu anachroniques pour son temps!). On y apprend cette fois comment la Moldavie a été peuplée au nord, par des Russes (il s'agit de la région de Bucovine), et au sud, par des Roumains.

L'histoire raconte qu'après avoir tué l'aurochs, les chasseurs du Maramures, sur le chemin du retour, rencontrent un vieil homme qui garde ses ruches. Interrogé sur le lieu qu'il habite et sur qui en est le maître, l'ermite révèle qu'il est russe, que le pays est "désert et sans maître "; "qu'il règne sur les bêtes sauvages et les oiseaux »; il leur apprend encore que le pays s'étend vers le bas jusqu'au Danube, et vers le haut jusqu'au Nistru, faisant la frontière avec le pays polonais, et que c'est un très bon endroit pour se nourrir. Alors, les chasseurs se hâtent d'amener leurs gens et de fonder la Moldavie. Et le vieux russe se dépêche lui aussi d'aller chercher les siens qu'il ramène dans la région de Suceava, Siret, Botosani. C'est ainsi que rapidement, les Roumains se sont étendus vers le bas et les Russes vers le haut $(. . .)^{38}$

\section{Quand l'Identité devient une affaire d'État ...}

A travers le jeu de miroirs qui s'instaure entre ces récits et ethnonymes, nous voyons se construire un écart différentiel, sur le modèle extrémiste du rapport antithétique de Soi à l'Autre. Le partenaire gênant est rabaissé au rang d'être vil, méprisable, tout proche de l'animal sauvage ou du monstre inhumain.

49 C'est bien ce mécanisme qui est à l'oeuvre dans les récits bulgares et hongrois sur les Roumains/Valaques: l'écart volontairement démesuré entre soi et les populations voisines résulte ici de l'origine délinquante qu'on leur prête ; ainsi justifie-t-on également leur mise à distance : il s'agit de les isoler de la société étatique dont ils risquent de bafouer les règles. Pour ce faire, les deux récits utilisent le même motif de la «terre désertique " située au-delà de ses frontières septentrionales: c'est l'au-delà danubien pour les Valaques expulsés par le Prince bulgare; et c'est l'au-delà de la chaîne montagneuse du Maramures, pour les Roumains qu' «installe» le Prince hongrois. Mais dans le cas des bergers récalcitrants, il s'agit de punir les exactions contre l'Etat: c'est pourquoi les Valaques sont condamnés à un retour à la sauvagerie jusqu'à ce qu'intégration s'en suive (religieuse compris !).

50 Dans le cas des futurs "Maramuresani », au contraire, il s'agit bon gré, mal gré, de récompenser ces anciens bandits puisqu'ils ont "servi l'Etat « : dès lors, leur exil prend la forme d'une véritable colonisation de nouvelles terres qui leur sont soigneusement 
assignées à chacun, avec des consignes précises quant à «l'aménagement du territoire », comme le récit y insiste :

«Ils (les Hongrois) les ont amené là-bas et ont réparti les bornes et les propriétés terriennes et les endroits pour (établir) les villages et les marchés ... »(Bratianu: 267).

Quant aux marques qui doivent servir à distinguer ces peuples récalcitrants, elles concernent toutes deux une "manipulation du poil», mais occupent des positions inversées d'un récit à l'autre, les premiers se retrouvant « poilus et velus »; les seconds étant au contraire, tonsurés.

52 A ce mouvement de bas en haut, qui caractérise ces motifs de l'exil et de l'isolement, propre à une mythologie "exogène », répond un mouvement de haut en bas : c'est la « descente » mythico-historiographique, « endogène » cette fois, des glorieux fondateurs d'États de la Roumanie.

Lorsque surgit une volonté centralisatrice, les Chroniques et Annales apparaissent à leur tour: elles sont chargées de relater les péripéties du peuplement des origines à la manière des mythes, surqualifiant les uns, disqualifiant les autres. "Si dans les sociétés sans Etat, écrit M. Carrin-Bouez (1985), le mythe tient souvent lieu d'histoire, lorsque l'Etat devient centralisateur, il impose son propre mythe qui devient référent obligé de toute histoire ».

L'ensemble des récits étiologiques sur les Valaques de tout poil et sur les Fondations d'Etats, s'accordent bien avec cette formulation, surtout si l'on entend ici le mythe dans sa dimension plurielle: des récits multiples qui s'éclairent mutuellement en se complétant dans un jeu d'oppositions, de ressemblances, voire de contradiction. Un jeu narratif qui disparait lorsque l'Etat centralisateur impose son mythe unique .

Ainsi se dessine peu à peu, entre mythe et histoire, aux frontières imprécises des jeunes Etats, la carte mouvante d'une géographie humaine où évoluent sans cesse des entités ethniques qui se distinguent et s'affrontent, investissent des territoires, s'en excluent ou s'y intègrent. Au diable ne plaise qu'à force de se raconter pareilles histoires, celles-ci ne finissent par servir de fondement aux génocides et fratricides de demain, si tant est qu'ils n'aient déjà commencé à servir de tels desseins mortifères ....

\section{BIBLIOGRAPHIE}

Benveniste, E., 1969, Le vocabulaire des institutions indo-européennes, vol. 1 : économie, parenté, société. Paris, Éditions de Minuit.

Bloch \& Von Wartburg, W. (1975), Dictionnaire étymologique de la langue française. Paris, PUF.

Bouissac, P. ,1971, « Pour une analyse ethnologique des entrées de clowns ». in : Ethnologie

Française, nouvelle série, T.1, n 3-4, 1971, pp. 7-17.

Carrin-Bouez, M., 1985, Éditorial à« Mythe et histoire », Cahiers de Littérature Orale, 1985, n 17 :

11.

de Saussure, F. (1916), Cours de Linguistique Générale. Paris, Payot 1982 
Eliade, M., 1970, « Le Prince Dragos et la chasse rituelle ». in : De Zalmoxis à Gengis Khan. Paris, Payot, pp. 131-161.

Gaignebet, CL., 1990, « La folle journée de Figaro : entre poil et jardinet ». in : Ethnologies d'Europe : Plantes et saisons ; calendriers et représentations. Les correspondances de Civilisations, Bruxelles.

Picoche, J., 1983, Dictionnaire étymologique du français. Paris ( Les Usuels de Robert).

Seton-Watson,R.W., 1937.Histoire des Roumains. De l'époque romaine à l'achèvement de l'unité. Paris, PUF.

Sorman, G.,1989, Les vrais penseurs de notre temps. Paris, Flammarion.

Ouvrages en roumain

Armbruster A., 1972, Romanitatea Romànilor. Bucuresti, Ed. A.R.S.R. (La roumanité des Roumains)

Bratianu, G.I.. 1980, Traditia istorice despre Întemeirea statelor romànesti. Bucuresti, Editura Eminescu. (Tradition historique sur la Fondation des Etats roumains)

Breban, Vasile, 1987, Dictionar general al limbii romàne. Buc., ed. stiintifica si enciclopedica, 1987. (Dictionnaire général de la langue roumaine)

Cartojan, N., (1980), Istoria literaturii romàne vechi. Buc., ed. Minerva. (lere ed. 1940-45) (Histoire de la littérature roumaine ancienne).

Densusianu, N., 1913, Dacia preistorica. Bucuresti, Institutul de arte grafic « carol Göbl » S-sor Ioan St Rasidescu. (La Dacie préhistorique)

Densusianu, N.,1966, Dictionarul Limbii Romàne. Bucuresti, Ed. A.R.S.R.

Hasdeu, B.P., (1879), Cuvente den Batrîni. Bucuresti, Ed. Didactica si Pedagogica, 1983. Tomul I. Limba romànà vorbità între 1550-1600. (Propos des Anciens : La langue roumaine parlée entre 1550-1600)

Hasdeu, B.P.,1982, Izvoarele istoriei României. (Vol. IV. Scriitori si acte bizantine secolele N-XV). Bucuresti, Ed. A.R.S.R. Publié par Haralamvie Mihàelescu, Radu Làzàrescu, Nicolae-Serban Tanasoca, Tudor Teoteoi, (Sources de l'Histoire de la Roumanie. vol. N. Ecrivains et actes byzantins du IV $-\mathrm{XV}$ es.)

Papahagi, T., (1981),Grai, folclor, etnografie. Bucuresti, Ed. Minerva. (Parler, folklore, ethnographie).

Stroescu, S., 1969, La typologie bibliographique des facéties roumaines. 2 vol. Burcuresti, Editions de l'A.R.S.R.

Référence des textes en bulgare

Sbomik za narodni umotvorenija (Recueil des Traditions Orales), Sofia, n 9 (1893) p. 127 et pp. 128-9.

Archives de Tsepenkov, Sofia, Institut d'Ethnographie.

Deux récits étiologiques collectés par S. Vaitov : L'origine des Tsiganes et des Valaques et Les Valaques velus. 


\section{NOTES}

1. Propos recueillis par Gui Sorman in: "Les vrais penseurs de notre temps": 129. La bibliographie des ouvrages cités se trouve en fin d'article.

2. Picoche: 83 .

3. Bloch \& von Wartburg

4. Voir à ce propos H. de Valencienne, ed. Wailly :338 (cité par Bràtianu).

5. Benveniste, op.cit. vol. 1

6. Voir à ce propos le symbolisme de l'ours poilu-homme sauvage que l'on rase en carnaval (Gaignebet : 1990) et la figure de saint André l'Oursier, dans le calendrier orthodoxe (Mesnil \& Popova, 1992, à paraître).

7. a.Voir le reportage :" Front National : la nébuleuse" de J. Cotta et $P$.

Martin, diffusée lors de l'émission télévisée "Envoyé Spécial" du 5 novembre 1992.

b. C'est encore dans cette méme perspective qu'il faut comprendre le sens du couple célébre des numéros de clowns de nos cirques, si bien analysés par P. Bouissac: les ressorts du comique y mobilisent tous les régistres de cette opposition entre un grotesque hirsute (le Gugus) et un glabre prétentieux qui dissimule sa pilosité sous une calotte blanche et lisse.

8. En ce qui concerne les multiples graphies utilisées pour transcrire ce terme, on se référera aux règles de translittération suivantes. A partir des langues slaves, la consonne uvulaire sourde (x) est notée hl khi chi $\mathrm{x}$. Ainsi, le méme vocable vlax pourrait être noté comme vlaah, vlax, vlakh etc. Ce phonème est noté $\mathrm{X}$ en alphabet grec et $\mathrm{X}$ en cyrillique. Nous avons opté pour la translittération de la consonne uvulaire sourde $\{\mathrm{x}\}$ comme $x$.

9. Benveniste, op.cit. vol. $1: 355-361$.

10. Ces populations se trouvent exclusivement dans les régions des Balkans, au sud du Danube ; ce sont de grands nomades et éleveurs qui pratiquent la transhumance à travers toute la Péninsule. Jadis, ils n'avaient pas de citoyenneté, à l'instar des Tsiganes.

11. "La présence de pasteurs valaques en Chalcidique est attestée notamment dans un manuscrit de 1105 : ils y sont signalés sous le règne de Alexie 1 Comnenul; on y mentionne le commerce qu'ils y entretiennent avec les Monastères du Mont Athos, et même le scandale qui serait né du fait que des femmes valaques se seraient infiltrées sur le territoire saint, déguisées en hommes pour y pratiquer quelque commerce parfois moins honnête que celui des produits de la bergerie... ( Voir Bratianu : 55) .

12. Les premières références du terme Romàn se trouvent dans les écrits de l'Empereur byzantin Constantin VII Porfirogène, datant du milieu du $\mathrm{X}^{\mathrm{e}}$ siècle. Bien que figurant dans un texte d'origine étrangère à cette population, le terme y apparaît comme un ethnonyme endogène. (Voir à ce propos Armbruster, p. 17 et suiv.).Nous remercions ici M. Nasta de nous avoir fourni cette référence.

13. Dans la mesure où il s'agit précisément d'une région de plaine, on suppose que cette désignation a été conservée par les populations qui sont venues des régions montagneuses (Seton-Watson : 28) Voir aussi plus loin, le thème de la "descente" (descalecat).

14. Précision de Hasdeu, Cuv.I : 287 et 535 .

15. a. Que l'on pense également à l'expression dépréciative qui avait cours dans les pays de langue française, avant-guerre: "moldo-valaque" était synonyme de cette autre expression injurieuse : "paysan du Danube".

b.Tout ceci n'empêche pas la petite minorité valaque (vlasi) résidant actuellement au nord-ouest de la Bulgarie de refuser énergiquement d'être "traitée" de Roumains: "Nous sommes des Valaques (Vlasi), nous n'avons rien à voir avec les Roumains (Rumancil d'au-delà du Danube" (Popova : Notes d'enquêtes en Bulgarie, août 1990) 
16. On notera que le a- du mot "aroumain" (aroman ) n'est pas un "a" privatif du terme "roumain" comme on pourrait s'y attendre. Il s'agit de la forme dialectale de ce mot qu'utilisent ces populations pour se désigner elles-mêmes.

17. Dans un manuscrit de 1308 dû à un moine anonyme, franciscain ou dominicain, il est signalé que "Entre la Macédoine, l'Achaia et Salonique, se trouve un certain peuple très nombreux et étendu appelé Vlahi, qui ont été autrefois pasteurs des Romains .... " (in Bratianu :68). D’une manière générale, l'historiographie a consigné les traces de ces populations de bergers "valaques ou roumains" sous l'appellation latine de "pastores Romanorum"( Voir à ce propos Armbruster, p. 28 et suiv.)

18. Rappelons cependant que l'éminent spécialiste des Aroumains, T. Papahagi, met en doute le fait que, d'un point de vue historique, ceux-ci n'aient pas connu l'agriculture; il s'appuie pour cela sur des arguments linguistiques en montrant que le vocabulaire des activités agricoles est. tout comme en roumain, d'origine latine. Ce qui permet de supposer qu'ils aient pu abandonner ces occupations pour se spécialiser dans le pastoralisme transhumant (Papahagi : 1981 :24)

19. Les deux vocables sont indubitablement allogènes, l'un (Vlas(l1 étant d'origine slave, l'autre (Vlax, Vlasi- cf. supra) - d'origine germanique. La transformation du phonème * $\mathrm{x}$ du mot vlax en *s au pluriel obéit à une loi phonologique des langues bulgare et serbo-croate stipulant que le phonème $*_{x}$ devant un $*_{i}$ se transforme en ${ }^{*}$ s.

20. Signalons cependant que le terme Vlax au singulier ne donne aucune prise à cette assimilation au vocable vlas, c'est-à-dire "poil/ cheveu".

21. Pour F. de Saussure (Cours p. 239/283) comme pour les anciens folkloristes de l'école de Max Müller, les associations et les rapprochements de type "étymologie populaire" apparaissent comme des jeux d'analogie fortuites fondés sur des homophonies accidentelles.

22. cf. SBNU 9, $1893: 128-9$

23. Il s'agit de la forme impérative du roumain Opresti ! qui signifie Arrête !

24. naphora, en bulgare, nafurà ou anafurà en roumain, du grec anaphora: c'est, dans le rite orthodoxe, l'équivalent de l'hostie catholique ; mais il s'agit ici de pain levé et non azyme.

25. Rappelons que le Royaume bulgare danubien fut fondé en 681 par une société de guerriers nomades d'origine turco-mongole. Ces Protobulgares aux yeux bridés étaient largement minoritaires par rapport aux populations conquises: Thraces et Slaves, essentiellement, mais aussi Valaques trans- et cis-danubiens.

26. Les souverains bulgares ne sont ni les premiers, ni les derniers à avoir échoué dans leurs aspirations colonisatrices vis-à-vis des populations de ces régions. Les Romains (entre le 1er et Ve Siècles) et les Turcs (du XIVe au XVIII Siècle), avaient également essayé de les vassaliser en en faisant de bons sujets disciplinés et soumis.

27. En roumain, comme dans d'autres langues qui ont gardé de telles figures archaïques, le terme miezànoapte, forme archaïsante de miezul noptii, littéralement "minuit", est aussi utilisé pour désigner le nord, à l'instar du "midi" indiquant le sud). Voir : Dictionarul Limbi Romane (DLR), T.VI, Bucuresti, Ed. A.R.S.R., 1966. (Communiqué par M. Nasta)

Que l'on pense aussi au mythe antique des Hyperboréens dont on trouve une curieuse actualisation au début de ce siècle, dans une oeuvre du folkloriste roumain N. Densusianu : Dacia preistorica (La Dacie préhistorique), Bucuresti, 1nstitutul de arte grafice "Carol Gôbl",1913.

28. Voir B.P. Hasdeu, Cuvente .. II : 152. Le texte cité fait partie d'une légende biblique apocryphe, "Legenda lui Avram" dont le prototype est écrit en grec et dont la version roumaine est traduite du slavon. On ne s'apesentira pas sur l'interprétation "patriotique" que fait Hasdeu de ce motif du chat! Notons que nous avons sans doute affaire ici à l'un de ces exemples qui marque l'interférence entre des sources écrites anciennes et des productions de la culture populaire orale.

29. L'anecdote est la suivante : Un Roumain va chez un marchand juif et lui demande un chat pour labourer ses terres "pour hue mais pas pour dia", parce qu'il n'a pas de boeufs. Un pari est fait entre le Juif et le Roumain: s'il lui donne un chat pour "dia", le Juif perd son pari et 
inversément. Lorsque le Roumain, après avoir attelé le chat à la charrue, lui crie "dia", le chat s'enfuit ... (in Stroescu, II : 1462 ,n05586).

30. Simion Dascalul n'est autre que l'un des copistes de la célèbre Chronique de Grigore Ureche : l'épisode cité ici constitue une ajoute que son auteur signale lui-même : "Moi, Simion Dascalul, je l'ai rédigé de la chronique hongroise .. "(in : Cartojan :279)

31. voir les textes reproduits en annexes de l'ouvrage de Bratianu (op. cit.) ainsi que les résumés et commentaires de Cartojan (id.)

32. Voir à ce propos la note 34

33. Au sud-ouest du Maramures, faisant la frontière avec la Moldavie.

34. Cartojan signale qu'il s'agit d'une coutume très ancienne du Maramures attestée dans un décret de saint Etienne. (op. cit. :279).

35. On s'étonnera à ce propos que des auteurs aussi érudits qu'un Cartojan ait eu aussi peu de distance critique pour considérer de tels textes dont la valeur exceptionnelle réside précisément dans le fait de nous donner accès à cette dimension mythique du récit. Ainsi notre précieux scribe se voit accusé par Cartojan d'inclusion d'une "note infamante sur l'origine des Roumains ... " où "la dignité du sentiment national est amortie ... "( cf p. 276 et 278). Que l'étude de la mythologie a donc besoin du "regard éloigné" !

36. Ce personnage également appelé Negru Voda,( en français, Rodolphe le Noir) est tenu pour le premier voïvode de Valachie et le fondateur des villes de Cîmpulung et de Curtea de Argès. Bien qu'attestée plus tardivement dans les textes que celle de Moldavie, on situe cette "descente" vers 1290, soit environ un demi-siècle avant celle de Dragos.

37. Sur l'universalité du thème de la chasse rituelle à l'origine d'une fondation, voir l'article de M. Eliade in : De Zalmoxis à Gengis Khan.

38. in Bratianu : 269.

\section{RÉSUMÉS}

Ethnic identities, as it is conceived by the relativist anthropological approach, is a symetric structure whose two constituent elements, the Same and the Other, are equivalent ontological identities. For traditional societies, the relation between constituent elements of this bipolar structure is by definition hierarchised: one is always superior or inferior to the other, the foreigner is far away of a neighbour. This asymetrical relation presupposes the existence of a precarious equilibrum of the relations between the autonomous ethnics groups engaged in mutually profitable relations. The text presented here analyses the identitary paradigm of several Sout- East European societies through two series of myths. The first serie is composed of exogenous etiologie myths, that is to say accounts on the origins of foreign ethnic groups: " hairy", or in other terms "uneducated" and "wild». The second serie is composed of endogenous accounts, obviously apologetic.

\section{INDEX}

Mots-clés : identité, ethnicité, Balkans, hiérarchie, altérité

Keywords : identity, ethnicity, hierarchy, alterity 


\section{AUTEURS}

MARIANNE MESNIL

Centre d'Ethnologie Européenne - Institut de Sociologie, Université libre de Bruxelles

ASSIA POPOVA

CNRS Lacito, Paris 